\title{
Chromosphere above sunspots as seen at millimeter wavelengths
}

\author{
Maria A. Loukitcheva ${ }^{1,2}$, Sami K. Solanki ${ }^{2}$ and Stephen M. White ${ }^{3}$ \\ ${ }^{1}$ Astronomical Institute, St.Petersburg University, Universitetskii pr. 28, \\ 198504 St.Petersburg,Russia \\ email: lukicheva@mps.mpg.de \\ ${ }^{2}$ Max-Planck-Institut für Sonnensystemforschung, D-37191 Katlenburg-Lindau, Germany \\ email: solanki@mps.mpg.de \\ ${ }^{3}$ Air Force Research Lab, Albuquerque, NM, 87117 USA \\ email: AFRL.RVB.PA@Hanscom.af .mil
}

\begin{abstract}
Millimeter emission is known to be a sensitive diagnostic of temperature and density in the solar chromosphere. In this work we use millimeter wave data to distinguish between various atmospheric models of sunspots, whose temperature structure in the upper photosphere and chromosphere has been the source of some controversy. From mm brightness simulations we expect a radio umbra to change its appearance from dark to bright (compared to the Quiet Sun) at a given wavelength in the millimeter spectrum (depending on the exact temperature in the model used). Thereby the millimeter brightness observed above an umbra at several wavelengths imposes strong constraints on temperature and density stratification of the sunspot atmosphere, in particular on the location and depth of the temperature minimum and the location of the transition region. Current $\mathrm{mm} / \mathrm{submm}$ observational data suggest that brightness observed at short wavelengths is unexpectedly low compared to the most widely used sunspot models such as of Maltby et al. (1986). A successful model that is in agreement with millimeter umbral brightness should have an extended and deep temperature minimum (below $3000 \mathrm{~K}$ ), such as in the models of Severino et al. (1994). However, we are not able to resolve the umbra cleanly with the presently available observations and better resolution as well as better wavelength coverage are needed for accurate diagnostics of umbral brightness at millimeter wavelengths. This adds one more scientific objective for the Atacama Large Millimeter/Submillimeter Array (ALMA).
\end{abstract}

Keywords. Sun: atmosphere, sun: chromosphere, sun: radio radiation, sun: sunspots

\section{Introduction}

Since the 1980s there have been several attempts to build a comprehensive semiempirical model of the atmosphere above a sunspot umbra. Deriving such models is a complicated process that tries to balance observations of a range of optical/UV lines (mostly formed in non-LTE conditions, see, e.g., Solanki (2003) and, when available, radio measurements of brightness spectra, together with ionization equilibrium and radiative transfer calculations that include heat transfer down from the corona as well as other factors (e.g. Fontenla et al. 1993). The radio data are particularly valuable because the measurements are in the Rayleigh-Jeans limit, meaning that measured brightness temperatures actually represent thermal electron temperatures in the optically thick atmosphere. The temperature at which a given frequency is optically thick (via thermal bremsstrahlung and, at very high frequencies, $H$ - opacity) is sensitive to density and temperature, and hence the radio data provide important constraints for modeling (e.g., Loukitcheva et al. 2004). However, while there are numerous radio temperature measurements of the 
quiet-Sun atmosphere, there are very few for sunspots, particularly at millimeter wavelengths, since good spatial resolution is required to isolate the sunspot temperature from its surroundings, and single-dish measurements generally do not have enough resolution. The highest resolution single-dish data, at 10-20 arcsec, are from JCMT (Lindsey \& Kopp 1995) and CSO (Bastian et al. 1993) but at submm, not mm, wavelengths. The sunspot umbra appears darker than the quiet Sun at these wavelengths. At millimeter wavelengths the instrument best suited for high resolution observations of sunspots is the 10-element Berkeley-Illinois-Maryland Array (BIMA) operating at $3.5 \mathrm{~mm}$ (Welch et al. (1996)). In this work we used BIMA observations of the temperature decrement above a sunspot umbra at $3.5 \mathrm{~mm}$, where model differences are large, to distinguish between various atmospheric models of sunspots.

\section{BIMA $3.5 \mathrm{~mm}$ observations}

On August 31, 2003 BIMA observed a small active region NOAA 10448 north of the solar equator. The images were reconstructed using the maximum entropy method (MEM) and restored with a Gaussian beam of $12 \operatorname{arcsec}$ (White et al. (2006)). The $3.5 \mathrm{~mm}$ image from BIMA is shown in Fig. 1 together with contemporaneous MDI photospheric magnetogram and BBSO CaII K images. The largest sunspot in the region is at the trailing (eastern) end of the region (see corresponding umbral and penumbral contours in Fig. 1). Its photospheric magnetic field does not exceed 2000 G. Clear depressions over this sunspot are seen both in radio and in calcium. The loop of bright emission encircling the sunspot in the CaII $\mathrm{K}$ image is essentially reproduced in the $3.5 \mathrm{~mm}$ image. From the BIMA maps we evaluate the " $3.5 \mathrm{~mm}$ umbra" to be approx. $400 \mathrm{~K}$ cooler than the Quiet Sun at this wavelength. Note that due to the use of MEM for image restoration we probably underestimate the umbral brightness decrement and $400 \mathrm{~K}$ is likely the lower limit for it. For comparison with the models we completed $3.5 \mathrm{~mm}$ brightness with the $\mathrm{mm}$ data at $0.35 \mathrm{~mm}, 0.85 \mathrm{~mm}$, and $1.2 \mathrm{~mm}$ from Lindsey \& Kopp (1995), plotted with circles in Fig. 2 (see next Section).
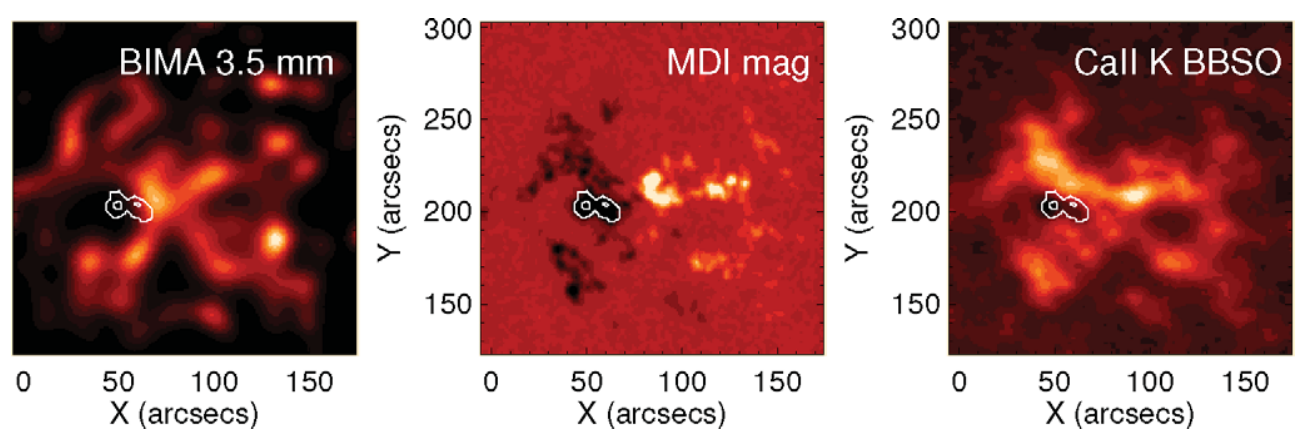

Figure 1. Active region NOAA 10448 observed on August 31, 2003. From left to right: BIMA image at $3.5 \mathrm{~mm}$, MDI photospheric magnetogram and BBSO CaII K image. The images were rotated to the same time and coaligned. White contours mark umbra and penumbra of the analyzed sunspot.

\section{Millimeter brightness spectra expected from sunspot models}

In order to distinguish the sunspot models, we have calculated the expected $\mathrm{sub} / \mathrm{mm}$ brightness temperatures at 24 selected wavelengths in the range $0.1-20 \mathrm{~mm}$. We analyzed 
classical sunspot models as well as their recent updates. These include the sunspot model of Avrett (1981), model $M$ of Maltby et al. (1986), the sunspot model of Severino et al. (1994), model $B$ of Socas-Navarro (2007), and model $S$ of Fontenla et al. (2009). The temperature versus height dependence of each of these models is shown in Fig. 2, together with the standard reference quiet-Sun atmosphere (model $C$ of Fontenla et al. (1993), commonly referred to as $F A L C)$. The calculations were done under the assumption
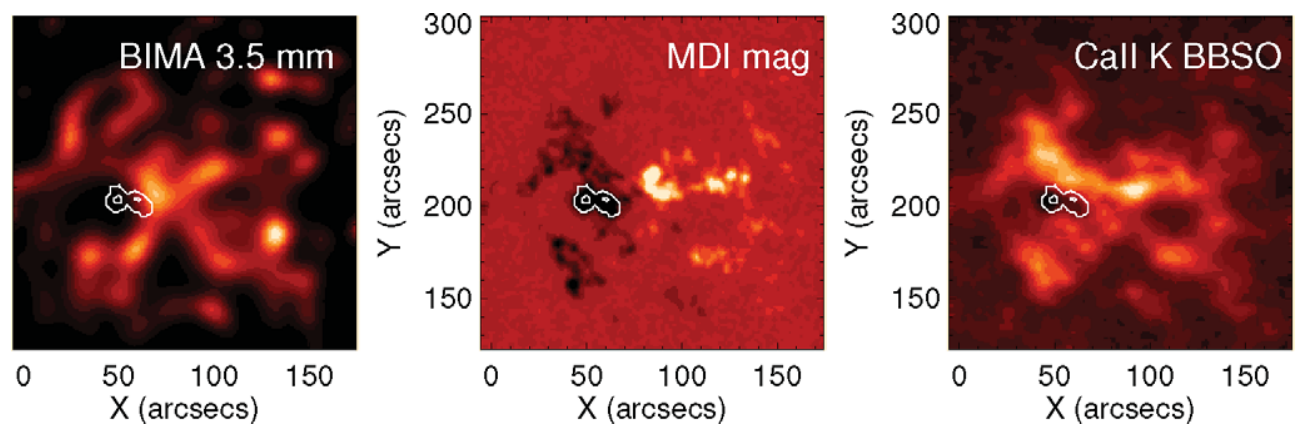

Figure 2. Left panel: The electron temperature as function of height in a number of standard models of the solar chromosphere above a sunspot, as labeled (see text). The solid black line is the reference quiet-Sun atmosphere known as FALC. Right panel: The brightness temperature as a function of wavelength for each model. Circles mark reported brightness temperature measurements

that bremsstrahlung opacity is responsible for the mm continuum radiation. We assume that gyroresonance opacity is negligible at these high frequencies. The expression for bremsstrahlung opacity in magnetic media was taken from Zheleznyakov (1996). The magnetic field was modelled by a vertical dipole buried under the photosphere according to Zlotnik (1968).

Figure 2 also shows the predicted brightness temperature spectra from each model, together with measurements culled from Lindsey \& Kopp (1995). The sunspot models differ significantly in the depth and extension of the temperature minimum region and in the location of the transition region. As a result they produce very different brightness spectra. One consequence of the models is that we expect the radio umbra to change its appearance from darker than the quiet Sun at short wavelengths to brighter at longer wavelengths. This turnover wavelength ranges from $6 \mathrm{~mm}$ in the case of Severino et al. (1994) to $0.3 \mathrm{~mm}$ in the Maltby et al. (1986) model. The model of Severino et al. (1994), which is the only model that has the turnover wavelength in agreement with the $3.5 \mathrm{~mm}$ measurements, is a modified version of the Maltby et al. (1986) and Caccin et al. (1993) models and is characterized by a steep temperature gradient in the photosphere, resulting in a minimum temperature of $2900 \mathrm{~K}$. On the whole most of the observational data points lie below the model spectra (Fig. 2). At short mm wavelengths the model of Fontenla et al. (2009) represents the observations relatively well, however longward of $3 \mathrm{~mm}$ the discrepancy between the observed and calculated brightness is substantial.

\section{Conclusions}

Millimeter brightness observations impose strong constraints on temperature and density stratifications of the sunspot atmosphere, in particular on the location and depth of the temperature minimum and the location of the transition region. Current $\mathrm{mm} / \mathrm{submm}$ observational data suggest that sunspot models, including the most widely used sunspot 
models of Maltby et al. (1986), the classical model of Avrett (1981), and recent models of Socas-Navarro (2007) and Fontenla et al. (2009), are too hot at chromospheric heights. A successful model that is in agreement with millimeter umbral brightness should have an extended and deep temperature minimum (below $3000 \mathrm{~K}$ ) as in the model of Severino et al. (1994). A new sunspot model by Avrett (this Proceedings) also possesses a very deep temperature minimum and therefore might result in reasonable agreement with the mm observations.

However, due to the spatial resolution limit of 12 arcsec we are not able to resolve the umbra cleanly in the present observations and the results obtained in this work are preliminary. A detailed study of the appearance of sunspot umbrae at $\mathrm{mm}$ waves requires substantially better resolution. Furthermore, good wavelength coverage is needed for accurate diagnostics of the turnover wavelength and hence successful modeling of the sunspot atmospheric temperature structure. We place high expectations on the Atacama Large Millimeter/Submillimeter Array (ALMA), which will start Early Science observations in 2011. With the instrument wavelength range of 0.3 to $10 \mathrm{~mm}$ and up to 64 antennas, ALMA will be an extraordinarily powerful instrument for studying the three-dimensional thermal structure of sunspots at chromospheric heights.

\section{References}

Avrett, E. H. 1981, in: L. E. Cram, J. H. Thomas (eds.), The Physics of Sunspots (National Solar Obs., Sunspot, NM), p. 235

Bastian, T. S., Ewell, M. W., Jr., \& Zirin, H. 1993, Astrophys. J., 415, 364

Caccin, B., Gomez, M. T., \& Severino, G. 1993, Astron. Astrophys, 276, 219

Fontenla, J. M., Avrett, E. H., \& Loeser, R. 1993, Astrophys. J., 406, 319

Fontenla, J. M., Curdt, W., Haberreiter, M., Harder, J., \& Tian, H. 2009, Astrophys. J., 707, 482

Lindsey, C. \& Kopp, G. 1995, Astrophys. J., 453, 517

Loukitcheva, M., Solanki, S. K., Carlsson, M., \& Stein, R. F. 2004, Astron. Astrophys, 419, 747

Maltby, P., Avrett, E. H., Carlsson, M., Kjeldseth-Moe, O., Kurucz, R. L., \& Loeser, R. 1986, Astrophys. J., 306, 284

Severino, G., Gomez, M. T., \& Caccin, B. 1994, in: R. J. Rutten, C. J. Schrijver (eds.), Solar Surface Magnetism (Dordrecht: Kluwer), p. 169

Socas-Navarro, H. 2007, Astrophys. J. Sup., 169, 439

Solanki, S. K. 2003, Astron. Astrophys. Rev., 11, 153

Welch, W. J., et al. 1996, Pub. Astr. Soc. Paci., 108, 93

White, S., Loukitcheva, M., \& Solanki, S. K. 2006, Astron. Astrophys, 456, 697

Zheleznyakov, V. V. 1996, Radiation in Astrophysical Plasmas

Zlotnik, E. Y.a. 1968, Soviet Astron., 12, 245 